\title{
DYNAMICAL BRAZOVSKII EFFECT
}

\section{S. Komura and N. Shimokawa}

Department of Chemistry, Graduate School of Science and Engineering, Tokyo Metropolitan University, Tokyo, Japan

\begin{abstract}
$\square \quad$ Based on Brazovskii's theory for fluctuation-induced first-order transition, we propose a dynamical model of ordered phases such as lamellar and hexagonal phases. Analysis of the suggested nonlinear equations shows that the system is bistable when the temperature is small enough. Close to the end point of the stable branch, the system can be transiently trapped in the intermediate ordered state.
\end{abstract}

Keywords Brazovskii effect, Block copolymers, Phase transition, Lamellar phase, Hexagonal phase, Amplitude equation

\section{INTRODUCTION}

Block copolymers are quite useful in many applications such as surfactants, compatibilizers in polymer blends, or adhesives. It is known that melts of block copolymers undergo microphase separation transition to produce various spatially ordered modulated phases (1). In 1980 Leibler obtained the phase diagram of block copolymer melts in the weak segregation limit within a mean-field theory (2). For a symmetric diblock melt for which the two blocks have the same length, the system is homogeneously disordered when temperature is higher than a critical value. Only at the critical point of the phase diagram, Leibler's mean-field theory predicts a second-order phase transition to a lamellar phase. On the other hand, asymmetric diblock melts undergo a first-order phase transition to a body-centered-cubic (BCC) phase by decreasing temperature. By further decreasing temperature, the mean-field theory suggests that there are first-order transitions from the BCC phase to a hexagonal phase and subsequently to the lamellar phase. Such a phase behavior coincides with

Received 10 January 2008; Accepted 24 March 2008.

Address correspondence to S. Komura, Department of Chemistry, Graduate School of Science and Engineering, Tokyo Metropolitan University, Tokyo 192-0397, Japan. E-mail: komura@tmu.ac.jp 
that obtained by Matsen and Schick according to numerical solution of self-consistent field equations without relying on any approximation (3).

However, the above mentioned phase behavior is modified when fluctuation corrections to Leibler's theory are taken into account. This fluctuation effect is called "Brazovskii effect" (4), which has been particularly calculated for diblock copolymers by Fredrickson and Helfand (5). They predicted that a symmetric diblock copolymer exhibits a first-order transition at a lower temperature than the second-order transition found within the mean-field theory. Moreover, the Brazovskii effect leads to windows in composition through which it is possible to pass from the homogeneous phase to each of the ordered phases by changing temperature. A similar phase diagram including the so-called "gyroid phase" was calculated before $(6,7)$. In the presence of Brazovskii critical fluctuations, a direct phase transition between the disordered and gyroid phases would be possible.

The onset of the ordering at finite values of wave vector occurs not only in block copolymers but also in other systems such as weakly anisotropic antiferromagnets (4), liquid crystals near the nematic-smectic C transition (8), and fluids near the Rayleigh-Bénard instability $(9,10)$. In all these systems, fluctuation-driven first-order transitions are expected to take place. Many efforts have been made to experimentally verify the Brazovskii effect in liquid crystal mixtures $(11,12)$, or in block copolymer melts (13-16).

Despite many investigations concerning equilibrium properties of Brazovskii effect, there are few works that deal with its dynamical aspects except those given in the references $(17-19)$. In the present article, we argue the kinetics of ordered phases by considering a dynamical version of Brazovskii effect within a coarse grained level. We propose nonlinear amplitude equations for the lamellar and hexagonal phases to discuss the time evolution of the respective ordered phases. We analyze the properties of the stationary solution of the amplitude equations, which are solved numerically. We find that the system is bistable when the temperature is low enough. In the next section, we first summarize the equilibrium properties of Brazovskii effect and present phase diagrams both for meanfield and Hartree approximations. In the third section, we present dynamical models for the lamellar and hexagonal phases. Analysis of the models and results of numerical simulations are given there. The final section concludes the article.

\section{EQUILIBRIUM PHASE BEHAVIOR}

We consider an order parameter $\phi(\mathbf{r})$, which vanishes on average in the homogeneous disordered phase, while it takes non-zero values in the ordered phases [see later Eqs. (2) and (3)]. Our starting point is the 
Landau-Brazovskii (LB) free energy functional, which can be written (in units of $\left.k_{\mathrm{B}} T\right)(2,5,20)$

$$
F[\phi(\mathbf{r})]=\int \mathrm{d} \mathbf{r}\left[\frac{\xi_{0}^{4}}{2}\left\{\left(\nabla^{2}+q_{0}^{2}\right) \phi(\mathbf{r})\right\}^{2}+\frac{\tau}{2}[\phi(\mathbf{r})]^{2}+\frac{\mu}{3 !}[\phi(\mathbf{r})]^{3}+\frac{\lambda}{4 !}[\phi(\mathbf{r})]^{4}\right],
$$

where $\xi_{0}$ is the correlation length, $q_{0}$ is the critical wavenumber, $\tau$ is the reduced temperature, $\mu$ and $\lambda$ are higher order vertex functions that are taken as constants (local approximation) (5). With the above mentioned free energy, the Fourier transform of the two point correlation functions shows a pronounced peak at $|\mathbf{q}|=q_{0}$. Hence the dominant fluctuations in the system are plane waves with wave vectors of magnitude $q_{0}$, or superpositions of such plane waves. The third order coefficient $\mu$ measures the intrinsic asymmetry, and vanishes for symmetric systems. Without loss of generality, one can assume that $\mu$ is positive because the LB free energy is invariant under the simultaneous transformation of $\phi \rightarrow-\phi$ and $\mu \rightarrow-\mu$. The fourth order coefficient $\lambda$ is positive for the stability reason.

\section{Mean-Field Approximation}

We first discuss the mean-field theory for the formation of the lamellar and hexagonal phases. For simplicity, we do not take into account the BCC phase nor the gyroid phase in this article. In the weak-segregation limit, the one-dimensional lamellar phase along the $z$-axis is expressed as

$$
\phi(\mathbf{r})=2 a_{\ell} \cos \left(q_{0} z\right),
$$

and the two-dimensional hexagonal phase in the $y$-z plane can be given by

$$
\phi(\mathbf{r})=2 a_{\mathrm{h}}\left[\cos \left(q_{0} z\right)+\cos \left(q_{0} \frac{\sqrt{3} y-z}{2}\right)+\cos \left(q_{0} \frac{-\sqrt{3} y-z}{2}\right)\right],
$$

where $a_{\ell}$ and $a_{\mathrm{h}}$ are the lamellar and hexagonal amplitudes, respectively. Inserting the above-mentioned forms of the order parameter in the LB free energy (1) and taking the average over one period, we obtain $(2,21)$

$$
F_{\ell}=\tau a_{\ell}^{2}+\frac{\lambda}{4} a_{\ell}^{4}
$$

for the lamellar phase and

$$
F_{\mathrm{h}}=3 \tau a_{\mathrm{h}}^{2}+2 \mu a_{\mathrm{h}}^{3}+\frac{15 \lambda}{4} a_{\mathrm{h}}^{4}
$$

for the hexagonal phase. By the minimization of these free energies and comparison of their respective values at the minimum, one can determine the phase transition lines between the disordered-lamellar, disorderedhexagonal, and hexagonal-lamellar phases. 
As shown in Fig. 1(a), the mean-field phase diagram can be represented in the $(\mu / \lambda, \tau / \lambda)$ plane. The disordered, hexagonal, lamellar, and inverted hexagonal phases are denoted by "D," "H," "L," and "IH," respectively. The phase diagram is symmetric with respect to $\mu=0$ as discussed before. When $\tau / \lambda$ is large and temperature is high, we have an homogeneous disordered phase. For symmetric cases with $\mu=0$, the system exhibits a second-order phase transition from the disordered phase to the lamellar phase at $\tau=0$. Hence the point $(\mu / \lambda$, $\tau / \lambda)=(0,0)$ is a critical point. For asymmetric cases with $\mu \neq 0$, both the disordered-hexagonal and hexagonal-lamellar transitions are firstorder, and the amplitude changes discontinuously at the transition line. By lowering temperature from the disordered phase, the hexagonal (or the inverted hexagonal) phase appears first at the line given by $\tau / \lambda=(4 / 45)(\mu / \lambda)^{2} \approx 0.089(\mu / \lambda)^{2}$. As the temperature is further lowered, then there is again a first-order phase transition from hexagonal to lamellar. The corresponding transition line is determined by the relation $\tau / \lambda=-[(7+3 \sqrt{ } 6) / 5](\mu / \lambda)^{2} \approx-2.87(\mu / \lambda)^{2}$.

\section{Hartree Approximation}

Brazovskii considered the role of the critical fluctuations on the phase transition that occurs in systems described by LB free energy (4). This can be done by the standard diagrammatic formalism, but attention must be paid to those integrals that diverge as $\tau$ vanishes. These divergences occur near the spherical shell $|\mathbf{q}|=q_{0}$. Brazovskii showed the way to
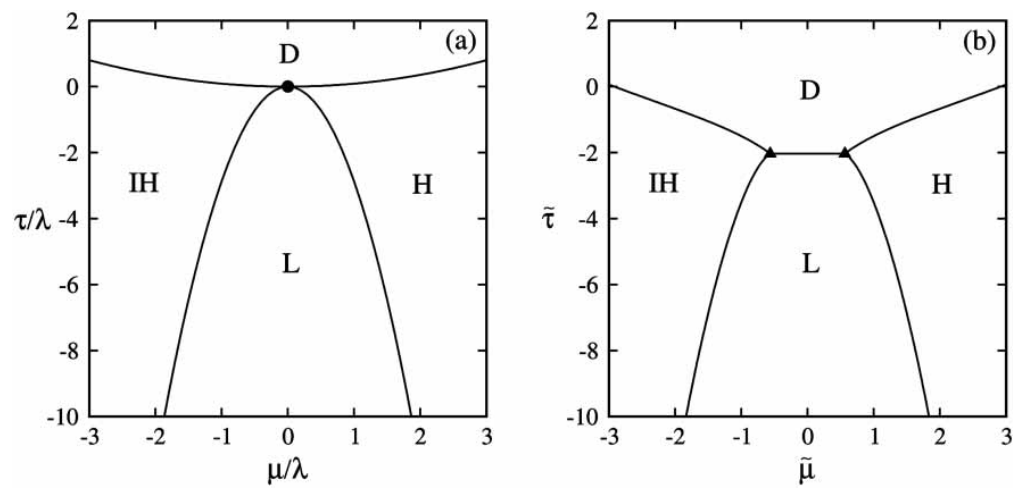

FIGURE 1 (a) Mean-field phase diagram of Landau-Brazovskii free energy functional (see Eq. (1)) as a function of $\mu / \lambda$ and $\tau / \lambda$. There are four different phases: the disordered phase (D), the hexagonal phase $(\mathrm{H})$, the lamellar phase $(\mathrm{L})$, and the inverted hexagonal phase $(\mathrm{IH})$. These phases are separated by first-order transition lines. The filled circle at $(\mu / \lambda, \tau / \lambda)=(0,0)$ indicates the critical point at which the transition is second-order. (b) Hartree phase diagram of Landau-Brazovskii free energy functional as a function of $\tilde{\mu}$ and $\tilde{\tau}$ defined in the text. The four phases are separated by first-order transition lines. The filled triangles at $(\tilde{\mu}, \tilde{\tau})=( \pm 0.564,-2.03)$ indicate the triple points. 
handle these divergences using a self-consistent Hartree approximation for the renormalized propagator. In this approximation, only a particular class of diagrams are summed in the perturbation series. The final result of Brazovskii's theory can be represented by thermodynamic potentials for the lamellar and hexagonal phases with respect to the disordered phase as given by

$$
\Phi_{\ell}\left(a_{\ell}\right)=\frac{1}{2 \lambda}\left(r_{\ell}^{2}-r_{\mathrm{d}}^{2}\right)+\alpha\left(r_{\ell}^{1 / 2}-r_{\mathrm{d}}^{1 / 2}\right)-\frac{\lambda}{4} a_{\ell}^{4}
$$

and

$$
\Phi_{\mathrm{h}}\left(a_{\mathrm{h}}\right)=\frac{1}{2 \lambda}\left(r_{\mathrm{h}}^{2}-r_{\mathrm{d}}^{2}\right)+\alpha\left(r_{\mathrm{h}}^{1 / 2}-r_{\mathrm{d}}^{1 / 2}\right)+2 \mu a_{\mathrm{h}}^{3}-\frac{3 \lambda}{4} a_{\mathrm{h}}^{4},
$$

respectively $(4,5)$, with a constant $\alpha=q_{0} /\left(8 \pi \xi_{0}^{2}\right)$ (20). In the above mentioned equations, the renormalized inverse susceptibilities of the disordered $\left(r_{\mathrm{d}}\right)$, lamellar $\left(r_{\ell}\right)$, and hexagonal $\left(r_{\mathrm{h}}\right)$ phases are determined by the following self-consistent equations $(4,5)$ :

$$
\begin{aligned}
& r_{\mathrm{d}}=\tau+\frac{\alpha \lambda}{r_{\mathrm{d}}^{1 / 2}}, \\
& r_{\ell}=\tau+\frac{\alpha \lambda}{r_{\ell}^{1 / 2}}+\lambda a_{\ell}^{2}, \\
& r_{\mathrm{h}}=\tau+\frac{\alpha \lambda}{r_{\mathrm{h}}^{1 / 2}}+3 \lambda a_{\mathrm{h}}^{2} .
\end{aligned}
$$

For our later purpose, we rescale the quantities as $\tilde{\Phi}_{\mathrm{i}} \equiv \lambda(\alpha \lambda)^{-4 / 3} \Phi_{\mathrm{i}}$, $\tilde{r}_{\mathrm{i}} \equiv(\alpha \lambda)^{-2 / 3} r_{\mathrm{i}}, \tilde{a}_{\mathrm{i}} \equiv \lambda^{1 / 2}(\alpha \lambda)^{-1 / 3} a_{\mathrm{i}}$ ("i" stands for either "d," " $\ell$," or "h"), and the Landau coefficients as $\tilde{\tau} \equiv(\alpha \lambda)^{-2 / 3} \tau, \tilde{\mu} \equiv \lambda^{-1 / 2}(\alpha \lambda)^{-1 / 3} \mu$.

As in the case of the mean-field theory, the phase diagram in the presence of critical fluctuations can be obtained by minimizing the above mentioned thermodynamic potentials and comparing their values at the minimum. The resulting phase diagram is presented in Fig. 1(b) on the $(\tilde{\mu}, \tilde{\tau})$ plane. The same phase diagram was previously presented in Ref. (21). Compared to the mean-field phase diagram shown in Fig. 1(a), there are two important differences. First the horizontal disorder-lamellar phase transition line is shifted to negative values of the temperature parameter at $\tilde{\tau} \approx-2.03$. Moreover, the transition is first-order even for symmetric cases with $\tilde{\mu}=0$. Hence a fluctuation-induced first-order transition takes place. Secondly, there exists a finite region of $\tilde{\mu}$ for which the hexagonal phase can never be stable. In fact the hexagonal phase appears only when the asymmetry is larger than $|\tilde{\mu}| \geq 0.564$. Notice that the three first-order transition lines meet at the triple point denoted by the filled 
triangles. For large values of $\tilde{\mu}$, the transition lines approach their meanfield values as given in Fig. 1(a).

\section{DYNAMICS OF ORDERED PHASES}

\section{Lamellar Phase}

In this section, we discuss the dynamics of the lamellar and hexagonal phases. Phenomenologically, we assume that the time evolution of the modulation amplitude is driven by the external force. For the lamellar phase, the proposed coarse grained amplitude equation has a relaxation form:

$$
\frac{d \tilde{a}_{\ell}(t)}{d t}=-\tilde{h}_{\ell}
$$

where the kinetic coefficient is taken to be unity, for simplicity, and $\tilde{h}_{\ell}$ is the external field defined by the derivative of the lamellar potential $\tilde{\Phi}_{\ell}$ with respect to the lamellar amplitude $\tilde{a}_{\ell}$ :

$$
\tilde{h}_{\ell}=\frac{1}{2} \frac{\delta \tilde{\Phi}_{\ell}}{\delta \tilde{a}_{\ell}}=\tilde{r}_{\ell} \tilde{a}_{\ell}-\frac{1}{2} \tilde{a}_{\ell}^{3}
$$

This is the Brazovskii's equation of state for the lamellar phase. In the above, the rescaled inverse susceptibility $\tilde{r}_{\ell}$ satisfies

$$
\tilde{r}_{\ell}=\tilde{\tau}+\frac{1}{\tilde{r}_{\ell}^{1 / 2}}+\tilde{a}_{\ell}^{2}
$$

according to Eq. (9). Eqs. (11), (12), and (13), describe the nonlinear time evolution of the lamellar amplitude $\tilde{a}_{\ell}(t)$. The only control parameter in the equation is $\tilde{\tau}$, corresponding to temperature.

We first argue the steady state condition (nullcline) of the abovementioned equation. This is equivalent to consider the zero field condition, that is, $\tilde{h}_{\ell}=0$. Then we have $\tilde{r}_{\ell}=\tilde{a}_{\ell}^{2} / 2$ from Eq. (12). Inserting this relation in Eq. (13), we obtain

$$
\tilde{\tau}=-\frac{1}{2} \tilde{a}_{\ell}^{2}-\frac{\sqrt{2}}{\left|\tilde{a}_{\ell}\right|}
$$

The above-mentioned equation is plotted in Fig. 2(a) in the $\left(\tilde{\tau}, \tilde{a}_{\ell}\right)$ plane. We see that the system is bistable when $\tilde{\tau}<-1.89$ (denoted by the filled circles). Notice that this value is larger than the equilibrium disorderlamellar transition temperature at $\tilde{\tau}=-2.03$ (see Fig. 1(a)). In Fig. 2(a), the stable branches are drawn by the solid lines. The dashed lines correspond to the threshold values of the initial amplitude $\tilde{a}_{\ell 0}$. The stationary state is the homogeneous disordered phase with $\tilde{a}_{\ell}=0$ 

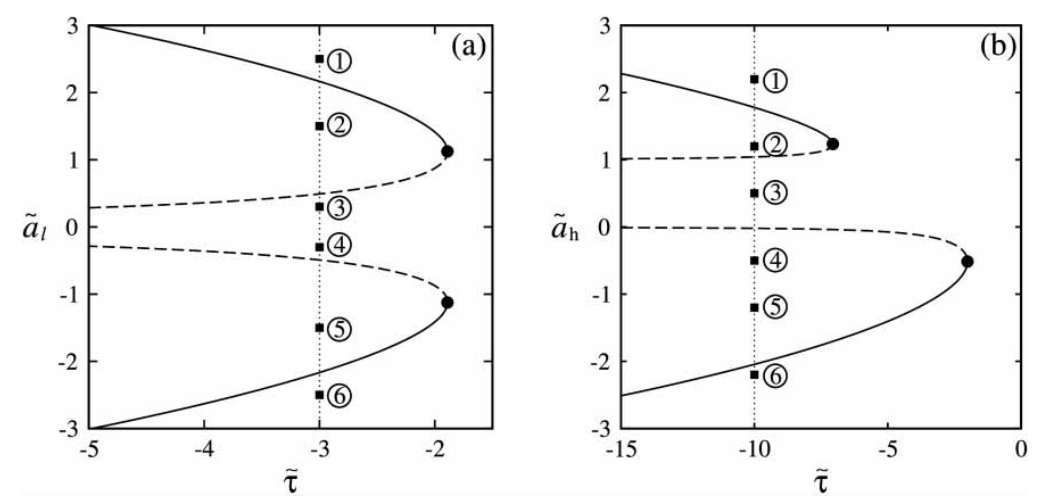

FIGURE 2 (a) Nullclines of the dynamic equation for the lamellar phase (see Eq. (11)) as a function of $\tilde{\tau}$ and $\tilde{a}_{\ell}$. The solid lines correspond to the stable branches, whereas the dashed lines give the threshold values of the initial amplitudes $\tilde{a}_{\ell 0}$. The filled circles at $\left(\tilde{\tau}, \tilde{a}_{\ell}\right)=(-1.89, \pm 1.12)$ indicate the end points of the stable branches. Six different filled squares at $\tilde{\tau}=-3$ are the initial values of $\tilde{a}_{\ell}$ used in the calculations of Fig. 3. (b) Nullclines of the dynamic equation for the hexagonal phase (see Eq. (15)) as a function of $\tilde{\tau}$ and $\tilde{a}_{\mathrm{h}}$. The asymmetry parameter is set to be $\tilde{\mu}=0.5$. The meaning of the solid and dashed lines is the same as in (a). The filled circles at $\left(\tilde{\tau}, \tilde{a}_{\mathrm{h}}\right)=(-2.01,-0.515)$ and $(-7.06,1.23)$ indicate the end points of the stable branches. Six different filled squares at $\tilde{\tau}=-10$ are the initial values of $\tilde{a}_{\mathrm{h}}$ used in the calculations of Fig. 5 .

when $\left|\tilde{a}_{\ell 0}\right|$ is smaller than these threshold values. In the opposite case, on the other hand, the system relaxes to the lamellar phase with $\tilde{a}_{\ell} \neq 0$. When $\tilde{\tau}>-1.89$, on the other hand, the disordered phase is always the stationary state.

In Fig. 3, we plot the time evolutions of the lamellar amplitude $\tilde{a}_{\ell}$ for six different initial values of $\tilde{a}_{\ell 0}$ while the temperature parameter is fixed to $\tilde{\tau}=-3$. The set of equations are solved numerically. Because different initial values of $\tilde{a}_{\ell 0}$ are realized at different temperatures, such a simulation corresponds to a temperature jump experiment in the lamellar phase. With this choice of parameter, the stationary value of the lamellar amplitude is $\left|\tilde{a}_{\ell}\right|=2.17$ (see the cross points of the solid and dotted lines in Fig. 2(a)). In all the cases, the lamellar amplitude changes monotonically and approaches to its stationary value. Compared to the relaxation to the lamellar phase, it takes much longer time to decay to the disordered phase with $\tilde{a}_{\ell}=0$. (Note that the time scale is different in Fig. 3(b).) In Fig. 4(a), we plot the time evolutions of the lamellar amplitude starting from the same initial value $\left(\tilde{a}_{\ell 0}=2\right)$ but with different temperature parameter values $\tilde{\tau}$. As mentioned earlier, the disordered phase is the stationary state when $\tilde{\tau}>-1.89$ and the amplitude $\tilde{a}_{\ell}$ decays to zero. For the values close to $\tilde{\tau}=-1.89$, however, the amplitude decreases in two steps. This means that the system is transiently trapped in the intermediate lamellar state. The trapping time becomes longer as the temperature is approached to $\tilde{\tau}=-1.89$. For large 

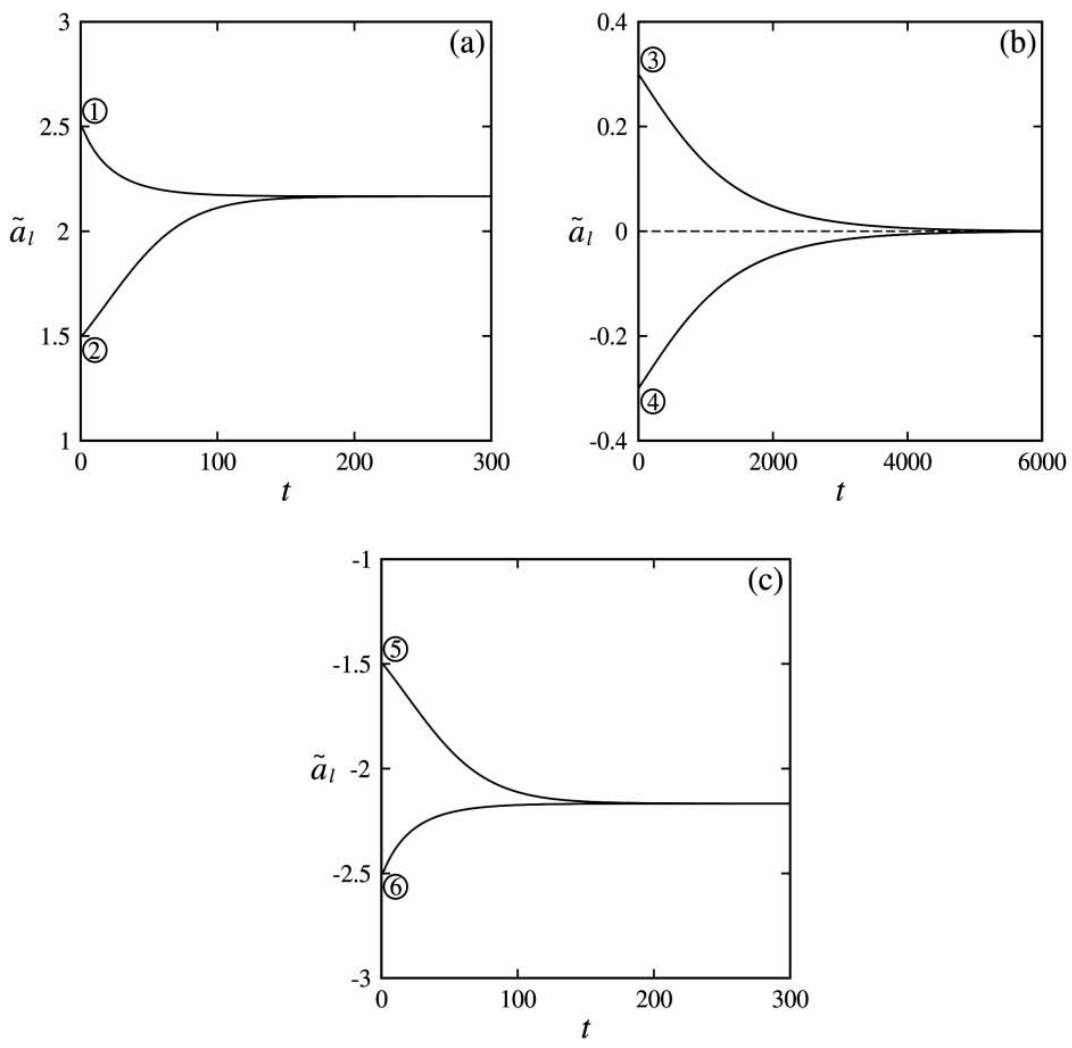

FIGURE 3 Time evolution of the lamellar amplitude $\tilde{a}_{\ell}$ for six different initial values $\tilde{a}_{\ell 0}$ shown in Fig. 2(a) when the temperature parameter is fixed to $\tilde{\tau}=-3$. The initial amplitudes are (a) $\tilde{a}_{\ell 0}=2.5$ and 1.5 , (b) 0.3 and -0.3 , (c) -1.5 and -2.5 . The steady state values are (a) $\tilde{a}_{\ell}=2.17$, (b) 0, (c) -2.17 .
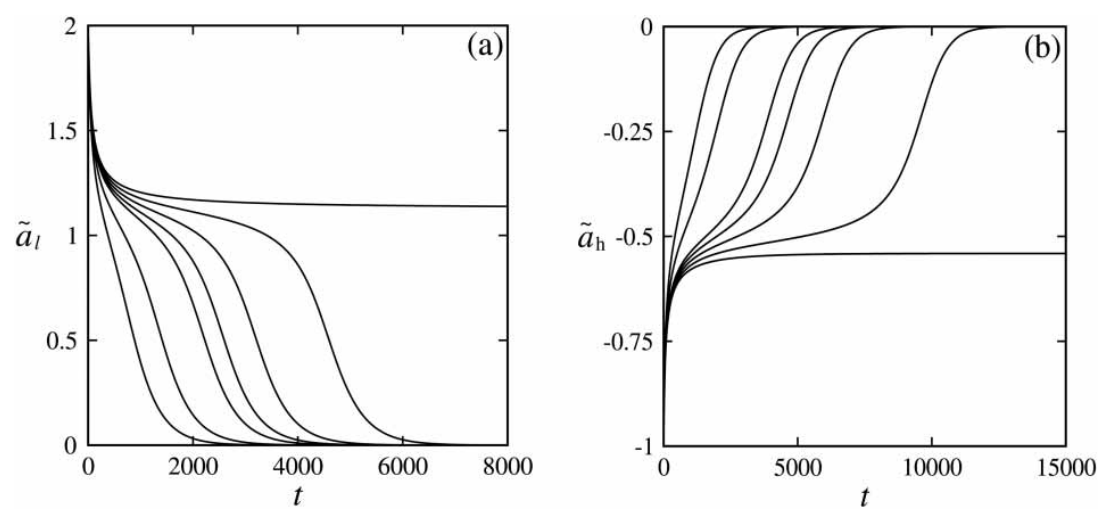

FIGURE 4 (a) Time evolution of the lamellar amplitude $\tilde{a}_{\ell}$ for different temperature parameters. The initial value is fixed to $\tilde{a}_{\ell 0}=2$. The temperature parameters are $\tilde{\tau}=-1.89,-1.88,-1.87,-1.86$, $-1.85,-1.8,-1.7$ from top to bottom. (b) Time evolution of the hexagonal amplitude $\tilde{a}_{\mathrm{h}}$ for different temperature parameters when $\tilde{\mu}=0.5$. The initial value is fixed to $\tilde{a}_{\mathrm{h} 0}=-1$. The temperature parameters are $\tilde{\tau}=-2.01,-2.00,-1.99,-1.98,-1.97,-1.90,-1.80$ from bottom to top. 
enough $\tilde{\tau}$ such as $\tilde{\tau}=-1.7$, the amplitude $\tilde{a}_{\ell}$ decays to zero almost monotonically.

\section{Hexagonal Phase}

Next we discuss the dynamics of the hexagonal phase. Similar to the case of the lamellar phase, the amplitude equation for $\tilde{a}_{\mathrm{h}}$ can be written as

$$
\frac{d \tilde{a}_{\mathrm{h}}(t)}{d t}=-\tilde{h}_{\mathrm{h}}
$$

where $\tilde{h}_{\mathrm{h}}$ is the external field given by

$$
\tilde{h}_{\mathrm{h}}=\frac{1}{6} \frac{\delta \tilde{\Phi}_{\mathrm{h}}}{\delta \tilde{a}_{\mathrm{h}}}=\tilde{r}_{\mathrm{h}} \tilde{a}_{\mathrm{h}}+\tilde{\mu} \tilde{a}_{\mathrm{h}}^{2}-\frac{1}{2} \tilde{a}_{\mathrm{h}}^{3} .
$$

This is again the Brazovskii's equation of state for the hexagonal phase. In Eq. (16) the rescaled inverse susceptibility $\tilde{r}_{\mathrm{h}}$ satisfies the relation

$$
\tilde{r}_{\mathrm{h}}=\tilde{\tau}+\frac{1}{\tilde{r}_{\mathrm{h}}^{1 / 2}}+3 \tilde{a}_{\mathrm{h}}^{2}
$$

from Eq. (10). Eqs. (15), (16), and (17) describe the time evolution of the hexagonal amplitude $\tilde{a}_{\mathrm{h}}(t)$. The control parameters are now $\tilde{\tau}$ and $\tilde{\mu}$.

The stationary state condition of $\tilde{a}_{\mathrm{h}}(t)$ is again the zero field condition $\tilde{h}_{\mathrm{h}}=0$, or equivalently, $\tilde{r}_{\mathrm{h}}=\tilde{a}_{\mathrm{h}}^{2} / 2-\tilde{\mu}_{\mathrm{h}}$. By substituting this relation into Eq. (17), we obtain

$$
\tilde{\tau}=-\frac{5}{2} \tilde{a}_{\mathrm{h}}^{2}-\tilde{\mu} \tilde{a}_{\mathrm{h}}-\frac{\sqrt{2}}{\left(\tilde{a}_{\mathrm{h}}^{2}-2 \tilde{\mu} \tilde{a}_{\mathrm{h}}\right)^{1 / 2}},
$$

when $\tilde{a}_{\mathrm{h}}^{2}-2 \tilde{\mu} \tilde{a}_{\mathrm{h}}>0$. The above-mentioned relation is plotted in Fig. 2(b) for $\tilde{\mu}=0.5$. When $\tilde{\mu} \neq 0$, the two nullclines become asymmetric. The meaning of the solid and dashed lines is the same as in Fig. 2(a). The two end points of the stable branches are located at $\left(\tilde{\tau}, \tilde{a}_{\mathrm{h}}\right)=(-2.01,-0.515)$ and $(-7.06,1.23)$. When the absolute value of the initial amplitude $\tilde{a}_{\mathrm{h} 0}$ is smaller than the dashed lines, the system decays to the homogeneous disordered phase with $\tilde{a}_{\mathrm{h}}=0$. The two dashed lines asymptotically approach $\tilde{a}_{\mathrm{h}}=0$ and 1 for small enough $\tilde{\tau}$.

In Fig. 5, we plot the time evolutions of the hexagonal amplitude $\tilde{a}_{\mathrm{h}}$ for six different initial values of $\tilde{a}_{\mathrm{h} 0}$ when $\tilde{\tau}=-10$ and $\tilde{\mu}=0.5$. With these parameters, the stationary values of the hexagonal amplitude are $\tilde{a}_{\mathrm{h}}=1.78$ and -2.05 . As in the case of the lamellar phase, the relaxation time for the disordered phase is longer than that for the hexagonal 



FIGURE 5 Time evolution of the hexagonal amplitude $\tilde{a}_{\mathrm{h}}$ for six different initial values $\tilde{a}_{\mathrm{h} 0}$ shown in Fig. 2(b) when the temperature and the asymmetry parameters are fixed to $\tilde{\tau}=-10$ and $\tilde{\mu}=0.5$, respectively. The initial amplitudes are (a) $\tilde{a}_{\mathrm{h} 0}=2.2$ and 1.2, (b) 0.5 and -0.5 , (c) -1.2 and -2.2 . The steady state values are (a) $\tilde{a}_{\mathrm{h}}=1.78$, (b) 0 and -2.05 , (c) -2.05 .

phase. In Fig. 4(b), we show the time evolutions of the hexagonal amplitude starting from the same initial value $\left(\tilde{a}_{\mathrm{h} 0}=-1\right)$ but with different values of $\tilde{\tau}$. For the values close to $\tilde{\tau}=-2.01$, the relaxation occurs in two steps so that the system is transiently trapped in the intermediate hexagonal state.

\section{CONCLUSION}

In this article we have discussed the dynamics of ordered phases by extending Brazovskii's theory for fluctuation-induced first-order transition. We have proposed self-consistent nonlinear equations both for the lamellar and hexagonal phases. Analysis of the stationary solutions of the equations shows that the system is bistable for small enough temperature parameter. This result has been confirmed by performing numerical simulation of the time evolution equations. Furthermore, we found that the system can be transiently trapped in the intermediate ordered state 
(either lamellar or hexagonal) close to the end point of the stable branch. These phenomena are expected to be observed in the future experiments.

\section{ACKNOWLEDGMENTS}

This work is supported by KAKENHI (Grant-in-Aid for Scientific Research) on Priority Areas "Soft Matter Physics" and Grant No. 18540410 from the Ministry of Education, Culture, Sports, Science, and Technology of Japan.

\section{REFERENCES}

[1] Hamley, I.W. (1998) The Physics of Block Copolymers; Oxford University Press: New York.

[2] Leibler, L. (1980) Macromolecules, 13: 1602.

[3] Matsen, M.W. and Schick, M. (1994) Phys. Rev. Lett., 72: 2660.

[4] Brazovskii, S.A. (1975) Sov. Phys. JETP, 41: 85.

[5] Fredrickson, G.H. and Helfand, E. (1987) J. Chem. Phys., 87: 697.

[6] Podneks, V.E. and Hamley, I.W. (1996) JETP Lett., 64: 617.

[7] Hamley, I.W. and Podneks, V.E. (1997) Macromolecules, 30: 3701.

[8] Swift, J. (1976) Phys. Rev. A, 14: 2274.

[9] Swift, J.B. and Hohenberg, P.C. (1977) Phys. Rev. A, 15: 319.

[10] Hohenberg, P.C. and Swift, J.B. (1992) Phys. Rev. A, 46: 4773.

[11] Brisbin, D., Johnson, D.L., Fellner, H., and Neubert, M.E. (1983) Phys. Rev. Lett., 50: 178.

[12] Martinez-Miranda, L.J., Kortan, A.R., and Birgeneau, R.J. (1986) Phys. Rev. Lett., 56: 2264.

[13] Bates, F.S., Rosedale, J.H., Fredrickson, G.H., and Glinka, C.J. (1988) Phys. Rev. Lett., 61: 2229.

[14] Bates, F.S., Rosedale, J.H., and Fredrickson, G.H. (1990) J. Chem. Phys., 92: 6255.

[15] Wolff, T., Burger, C., and Ruland, W. (1993) Macromolecules, 26: 1707.

[16] Koga, T., Koga, T., and Hashimoto, T. (1999) Phys. Rev. E, 60: R1154.

[17] Fredrickson, G.H. and Binder, K. (1989) J. Chem. Phys., 91: 7265.

[18] Cates, M.E. and Milner, S.T. (1989) Phys. Rev. Lett., 62: 1856.

[19] Koppi, K.A., Tirrell, M., and Bates, F.S. (1993) Phys. Rev. Lett., 70: 1449.

[20] Hohenberg, P.C. and Swift, J.B. (1995) Phys. Rev. E, 52: 1828.

[21] Marques, C.M. and Cates, M.E. (1990) J. Phys. France, 51: 1733. 